\section{Leishmaniasis cutánea y herpes zoster multidermatómico}

\section{Margarita Arboleda, Laura Jaramillo, Diana Ortiz y Alejandro Díaz.}

\section{Cutaneous leishmaniasis and multidermatomic herpes zoster}

Standard treatment of leishmaniasis consists of n-metilglucamine, meglumine antimoniate, which can trigger side effects such as general malaise, renal and hepatic impairment, and cardiac arrhythmias. Infrequently, reactivations of varicella-zoster virus infections have been reported. This paper describes a patient with cutaneous leishmaniasis in treatment with meglumine and herpes zoster multiplex. After ruling out other possible causes of immunosuppression, an acyclovir therapy was initiated.

Key words: Cutaneous leishmaniasis, herpes zoster, meglumine antimoniate, drug toxicity.

Palabras clave: Leishmaniasis cutánea, herpes zoster, antimoniato de meglumina, toxicidad de medicamentos.

\section{Introducción}

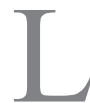

a leishmaniasis es una parasitosis tisular causada por protozoos del género Leishmania, la cual es considerada una antropozoonosis que afecta al hombre por la picadura de insectos flebótomos del género Lutzomyia. Las manifestaciones clínicas varían desde formas asintomáticas y lesiones cutáneas autorresolutivas, hasta el compromiso mucocutáneo y visceral con alta morbimortalidad. La forma cutánea corresponde a $95 \%$ de los casos. La úlcera crónica con bordes indurados asociado a adenomegalias es la forma más común de presentación en nuestro medio ${ }^{1}$.

La leishmaniasis es un problema de salud pública en Colombia, reportándose 8.365 casos nuevos de leishmaniasis cutánea (LC), 133 casos de la forma mucosa y 12 casos de la forma visceral durante el año $2012^{2}$.

El antimoniato de meglumina, que junto con el estibogluconato sódico son parte de los antimoniales pentavalentes, es el tratamiento de primera elección para todas las formas de la enfermedad en Colombia ${ }^{3}$. A pesar de su uso durante décadas, el mecanismo de acción de estos medicamentos no está completamente establecido y se cree que interfieren con diversos procesos bioenergéticos del parásito ${ }^{1}$

A pesar de que este medicamento tiene variados efectos adversos, desde leves como artralgias, mialgias, dolor en el sitio de aplicación, hasta graves como toxicidad hepática, pancreática, renal y cardíaca ${ }^{4,5}$ es, en general, bien tolerado por la mayoría de los pacientes y son reversibles al disminuir la dosis o suspender la terapia ${ }^{6,7}$.

Instituto Colombiano de Medicina Tropical. Apartadó, Colombia. (MA, $\sqcup)$ Universidad CES, Medellín, Colombia. (DO, AD)

No existe conflicto de interés entre los autores.

Financiamiento: Instituto Colombiano de Medicina Tropical y por la Universidad CES, Antioquia, Colombia.

Recibido: 18 de febrero de 2013 / Aceptado: 3 de octubre de 2013

\section{Correspondencia a:}

Margarita Arboleda.

marboleda@ces.edu.co
Existen escasos reportes de reactivación del virus varicela-zoster (VVZ) asociada al tratamiento con antimoniales, especialmente con el estibogluconato sódico, aunque también los hay con meglumina ${ }^{8,11}$. Hasta ahora no había comunicación de esta asociación en Colombia.

Se presenta el caso de un paciente con un herpes zoster multidermatómico asociado al tratamiento con meglumina por una LC.

\section{Caso clínico}

Mujer de 34 años, residente en el municipio de Carepa en el Urabá Antioqueño, Colombia y trabajadora rural en las veredas "El Cerro" y "Remedia pobre", áreas endémicas para leishmaniasis. Consultó por un cuadro de dos meses de evolución de pápulas eritematosas en el dorso de la mano izquierda con aumento progresivo de tamaño y posterior ulceración. No refirió otra sintomatología asociada, ni traumas o picaduras de insectos. Tenía el antecedente de varicela a los 9 años y hepatitis A y malaria por $P$. vivax a los 10 años.

La paciente fue evaluada en nuestro centro, encontrándose en buenas condiciones generales, con signos vitales estables. En el examen físico se encontró integridad de la mucosa nasal y en la piel se encontraron dos lesiones ulceradas limpias de $2 \mathrm{~cm}$ de diámetro, con bordes indurados, localizadas en el dorso de la falange proximal del cuarto dedo y el dorso de la mano izquierda, asociadas a una adenomegalia dolorosa de $1,5 \mathrm{~cm}$ epitroclear. Se realizó un examen directo de la lesión encontrándose la presencia de amastigotes de Leishmania spp., confirmándose el diagnóstico de LC.

Se prescribió tratamiento con meglumina i.m. $20 \mathrm{mg} / \mathrm{kg} /$ día, por 20 días. Previo al inicio del tratamiento se tomaron exámenes generales (uroanálisis, transaminasas hepáticas, amilasas, nitrógeno ureico, creatinina y hemograma) que fueron normales. La paciente fue derivada a su municipio de origen para la aplicación supervisada del tratamiento, con respuesta clínica adecuada, sin referir efectos secundarios. Tres días después de terminado el tratamiento, consultó por presentar lesiones papulares, vesiculares y pustulares con ardor y dolor local, inicialmente en la región submamaria derecha con extensión hacia el dorso en el dermatoma T6, y posteriormente en el tercio medio del antebrazo izquierdo y dorso de la mano izquierda en el dermatoma C8, que aparecieron a partir del día 15 de tratamiento con meglumina (Figuras 1 y 2). Se manejó como una reacción alérgica, con corticosteroides tópicos sin mejoría, por lo cual se remitió a nuestro centro en donde se realizó el diagnóstico de herpes zoster. Se realizó un test de Tzanck de una lesión vesicular que demostró la presencia de células gigantes multinucleadas, lo que apoyó el diagnóstico clínico. Se manejó con aciclovir oral, $800 \mathrm{mg}$ cinco veces al día, durante 7 días.

Se realizaron estudios de extensión: ecografía mamaria, citología vaginal, serología para sífilis y para infección por VIH, todos negativos. No se realizó hemograma de control ya que no tenía indicación clínica. No fue posible la confirmación microbiológica del VVZ ni serología. En el control médico a

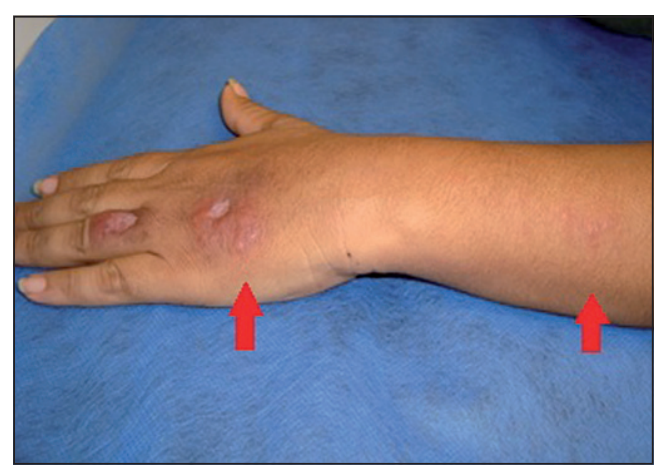

Figura 1. Lesiones vesiculares por VVZ alrededor de la cicatriz de leishmaniasis cutánea y en el tercio medio del antebrazo izquierdo (flechas rojas) dermatoma C8. 


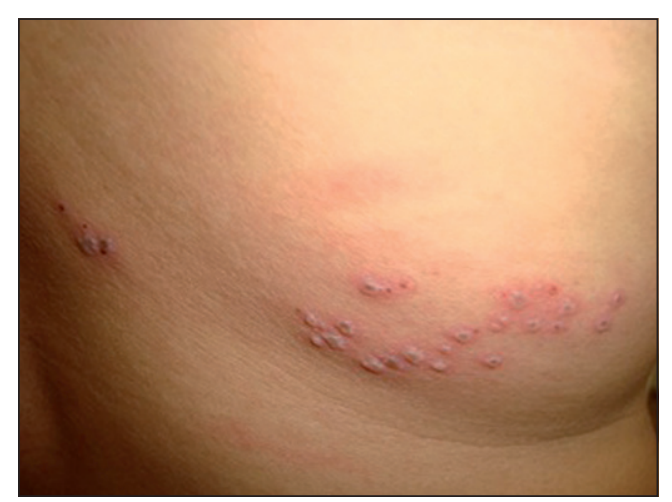

Figura 2. Lesiones vesículo-pustulosas por VVZ en el dorso, dermatoma T6.

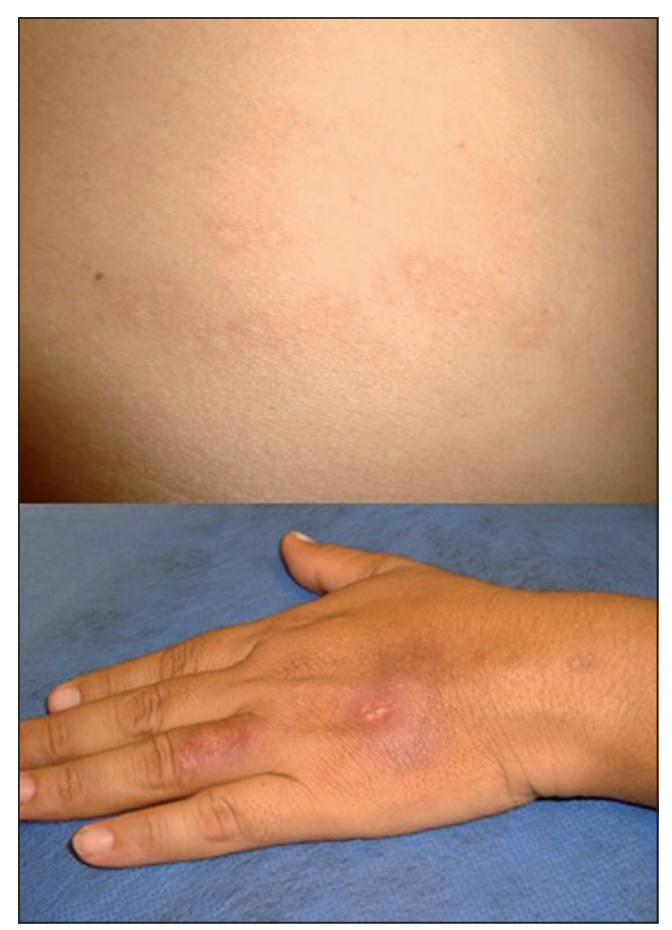

Figura 3. Lesiones hiperpigmentadas postinflamatorias por herpes zoster cicatrizado, dermatoma T6. Lesiones hipopigmentadas postinflamatorias por leishmaniasis cutánea curada en el dorso de la mano izquierda.

los seis meses no había aparición de nuevas úlceras de leishmaniasis ni de herpes zoster, con cicatrización adecuada de las lesiones antiguas (Figura 3).

\section{Discusión}

El caso presentado de herpes zoster multidermatómico, probablemente desencadenado por el tratamiento con meglumina en una paciente con LC, puede atribuirse a un efecto secundario de este medicamento ${ }^{13}$.

El test de Tzanck, aunque no es la prueba de oro, es un examen que apoya el diagnóstico del virus herpes, y ante un cuadro clínico característico, dado su bajo costo y facilidad, ayuda a establecer el diagnóstico definitivo ${ }^{14}$.

Los efectos secundarios de los antimoniales pentavalentes incluyen síntomas sistémicos como malestar general, mialgias, artralgias y dolor en el sitio de aplicación ${ }^{13,15}$. Otras alteraciones de mayor gravedad incluyen compromiso renal, hepático, cardíaco, pancreático ${ }^{1,15}$ y otras relacionadas con eventos idiosincráticos infrecuentes, como la neuropatía periférica sensorial reversible, asociada con la administración de estibogluconato ${ }^{16}$, hipersensibilidad alérgica medicamentosa ${ }^{17}$ e incluso la muerte ${ }^{18}$. Sin embargo, existe evidencia que a las dosis convencionalmente utilizadas (20 $\mathrm{mg} / \mathrm{kg} /$ día), la frecuencia de eventos adversos graves es baja y el perfil de seguridad y eficacia para ambos medicamentos es similar ${ }^{13,15}$.

A la fecha, no existe evidencia que sustente que la presencia de leishmaniasis en sí, sea un factor de riesgo para el desarrollo de herpes zoster o viceversa. Hay escasos reportes que describen la aparición de lesiones de LC y herpes zoster de forma simultánea en pacientes con infección por $\mathrm{VIH}^{19,20}$. Esto resalta el hecho de que la LC puede afectar piel previamente comprometida $^{19,20}$. Sin embargo, esto no justifica un nexo de causalidad para que una enfermedad desencadene la otra.

La reactivación de la infección por VVZ secundaria al tratamiento con antimoniales es un efecto secundario reportado escasamente en la literatura científica. Wortmann y cols. comunicaron tres casos de herpes zoster en 84 pacientes tratados con estibogluconato sódico, lo que representó una incidencia de 3,6\%, comparado con un $0,2 \%$ en personas sanas entre 20 y 30 años (18 veces mayor a la esperada en población sana). Los pacientes desarrollaron herpes zoster en un tiempo promedio de 23 días luego de iniciada la terapia (rango entre 14 y 50 días). Debido a que todos los casos ocurrieron durante o después del tratamiento, los investigadores sugieren que el medicamento pudo haber contribuido a la reactivación ${ }^{12}$. Llanos y cols., que investigaron el tratamiento con estibogluconato solo o en combinación con alopurinol para el tratamiento de leishmaniasis visceral, encontraron una incidencia similar. De los 81 pacientes que recibieron el antimonial, hubo tres casos de herpes zoster. $(3,7 \%)^{21}$. Otro reporte más reciente informó el caso de una paciente de 24 años, quien en el día 17 de tratamiento con estibogluconato presentó una meningitis aséptica y un rash vesicular en la región del dermatoma T1. La serología para VVZ fue positiva, así como la RPC para VVZ en LCR?.

Las publicaciones de casos asociados con meglumina también son escasos. Oliveira Neto y cols., en una serie de pacientes con leishmaniasis mucosa que recibieron meglumina, reportaron dos pacientes que desarrollaron herpes zoster y tres pacientes con herpes simplex al finalizar la terapia ${ }^{10}$. En otra serie se registraron, en pacientes hospitalizados con herpes zoster, dos casos $(3,8 \%)$ que habían recibido meglumina previamente ${ }^{11}$.

El mecanismo por el cual ocurre la reactivación del VVZ en pacientes que reciben antimoniales no está completamente dilucidado. Sin embargo, se sabe que los linfocitos T (LT) son necesarios para la inactivación del virus, y una disminución relativa de los LT CD4, que afecta la respuesta celular específica de memoria, favorece su reactivación. Esto sugiere que el uso de antimoniales produce una linfopenia que puede contribuir a la reactivación del $\mathrm{VVZ}^{8,11,12}$

Esto ha llevado a estudiar la respuesta linfocitaria durante el tratamiento con antimoniales. En un estudio prospectivo en ocho pacientes con LC y que recibieron estibogluconato sódico (de los cuales uno presentó un herpes zoster), se identificó una disminución de los LT totales, CD4 y CD8 con respecto al nivel basal, en el $7^{\circ}$ día de tratamiento. La disminución del recuento de CD4 fue en promedio de 67\% (306 céls $/ \mathrm{mm}^{3}$ ) con respecto al nivel basal. Los cambios en la relación CD4/CD8 y en el recuento de LB no alcanzaron significancia estadística. Luego de un período de uno a seis meses, el recuento de linfocitos estaba en el nivel pre-tratamiento. Se realizaron análisis de viabilidad celular in vitro, y se demostró que el estibogluconato sódico no era tóxico para los linfocitos. Los investigadores concluyeron que el medicamento disminuye transitoriamente las subpoblaciones de CD4 y CD8 por mecanismos aun no entendidos, pero que este efecto es transitorio ${ }^{12}$.

Otro estudio, evaluó a diez pacientes con diagnóstico de LC con tratamiento con estibogluconato. Se tomaron muestras de sangre al inicio y diez días después de iniciado el tratamiento y se realizaron análisis leucocitarios por citometría de flujo y serológicos para VVZ. No se documentó ningún 
caso de herpes zoster en un período de seis meses. Al comparar los resultados del día 0 con el día 10 de tratamiento, encontraron una disminución de los leucocitos totales, linfocitos totales, LT CD4, citotóxicos, de memoria y células natural killer. Los niveles de Ig anti VVZ no tuvieron cambios significativos ${ }^{8}$. En el estudio de Llanos y cols., también se evidenció que la toxicidad hematológica (anemia, leucopenia con linfopenia y trombocitopenia) asociada al estibogluconato era dependiente de la dosis ${ }^{21}$. Estos hallazgos soportan la teoría que el recuento de leucocitos, especialmente los LT CD4, disminuyen durante el tratamiento con estibogluconato, sin tener un efecto demostrable en la respuesta inmune celular contra el VVZ. Sin embargo, al disminuir la población total de leucocitos, se incluirían los específicos de memoria para VVZ lo cual podría explicar las reactivaciones, aunque esta teoría no ha sido aclarada aún ${ }^{8}$.

En el caso presentado, el recuento de leucocitos totales fue normal al ingreso. Desafortunadamente no se pudo determinar algún cambio en el recuento ya que no se realizó hemograma al finalizar el tratamiento, ni tampoco se evaluó el recuento de LT CD4.

Agradecimientos: Agradecemos al personal del ICMT de Apartadó, especialmente a la bacterióloga Luz Yaned Úsugay y a la paciente que facilitó el acceso a la historia clínica.

\section{Resumen}

El tratamiento estándar de la leishmaniasis con n-metilglucamina o antimoniato de meglumina puede desencadenar reacciones secundarias sistémicas como compromiso renal, hepático y trastornos del ritmo cardíaco. Se han descrito casos esporádicos de reactivación del virus varicela-zoster. Se describe el caso de una paciente con un herpes zoster multidermatómico desencadenado por antimoniato de meglumina (meglumina) para el tratamiento de una leishmaniasis cutánea. Después de descartar inmunosupresión el paciente fue tratado con aciclovir.

\section{Referencias bibliográficas}

1.- Restrepo M, Botero D. Leishmaniasis. Restrepo M, Botero D, ed. $5^{\circ}$ ed. Medellin, Colombia: Corporación para Investigaciones Biológicas, CIB; 2012.

2.- $\quad$ SIVIGILA. Boletín Epidemiológico Semanal $N^{\circ} 52$. Sistema Nacional de Vigilancia en Salud Pública. Instituto Nacional de Salud, Colombia, 2012. http://www.ins.gov.co/lineas-de-accion/Subdireccion-Vigilancia/sivigila/ Paginas/vigilancia-rutinaria.aspx: Fecha de acceso: 20 de enero 2013.

3.- INS. Guía para la atención clínica integral del paciente con leishmaniasis. Bogotá, Colombia. Instituto Nacional de Salud. Ministerio de la Protección Social, 2010. Disponible en: www.ins.gov.co. (Fecha de acceso: 20 de enero de 2013).

4.- Singh N, Kumar M, Singh R K. Leishmaniasis: current status of available drugs and new potential drug targets. Asian Pac J Trop Med 2012; 5: 485-97.

5.- Saldanha A C, Romero G A, Merchan-Hamann E, Magalhães A V, Macedo Ve O. A comparative study between sodium stibogluconate BP 88R and meglumine antimoniate in the treatment of cutaneous leishmaniasis. I. The efficacy and safety. Rev Soc Bras Med Trop 1999; 32: 383-7.
6.- Neves L O, Talhari A C, Gadelha E P, Silva Júnior R M, Guerra J A, Ferreira L C, et al. A randomized clinical trial comparing meglumine antimoniate, pentamidine and amphotericin $\mathrm{B}$ for the treatment of cutaneous leishmaniasis by Leishmania guyanensis. An Bras Dermatol 2011; 86: 1092-101.

7.- Minodier P, Parola P. Cutaneous leishmaniasis treatment. Travel Med Infect Dis 2007; 5: 150-8.

8.- Yoon IK, Cox J, Zhou Y, Lukes Y, Reinhardt B, Valencia-Micolta A, et al. Varicella zoster virus-specific immune response after treatment with sodium stibogluconate for cutaneous leishmaniasis. Am J Trop Med Hyg 2008; 78: 402-5.

9.- Hartzell J D, Aronson N E, Nagaraja S, Whitman T, Hawkes C A, Wortmann G. Varicella zoster virus meningitis complicating sodium stibogluconate treatment for cutaneous leishmaniasis. Am J Trop Med Hyg 2006; 74: 591-2.

10.- Oliveira-Neto M P, Mattos M, Pirmez C, Fernandes O, Gonçalves-Costa S C, Souza C F, et al. Mucosal leishmaniasis ("espundia") responsive to low dose of N-methyl glucamine (Glucantime) in Rio de Janeiro, Brazil. Rev Inst Med Trop Sao Paulo 2000; 42: 321-5.

11.- Maguiña C, Bazán L, Álvarez H, Gotuzzo E, Echevarría J, Seas Ramos C, et al. Estudio retrospectivo de pacientes hospitalizados por herpes zoster en el Hospital Nacional Cayetano Heredia entre los años 1980-1998. Folia Dermatol Peru 1999; 10: 19-31.

12.- Wortmann G W, Aronson N E, Byrd J C, Grever M R, Oster C N. Herpes zoster and lymphopenia associated with sodium stibogluconate therapy for cutaneous leishmaniasis. Clin Infect Dis 1998; 27: 509-12.

13.- Henao H H, Osorio Y, Saravia N G, Gómez A, Travi B. Efficacy and toxicity of pentavalent antimonials (Glucantime and Pentostam) in an American cutaneous leishmaniasis animal model: luminometry application. Biomédica 2004; 24: 393-402.

14.- Durdu M, Seçkin D, Baba M. The Tzanck smear test: rediscovery of a practical diagnostic tool. Skinmed 2011; 9: 23-32.

15.- Aronson N E, Wortmann G W, Johnson S C, Jackson J E, Gasser R A, Magill A J, et al. Safety and efficacy of intravenous sodium stibogluconate in the treatment of leishmaniasis: recent U.S. military experience. Clin Infect Dis 1998; 27: 1457-64.

16.- Brummitt C F, Porter J A, Herwaldt B L. Reversible peripheral neuropathy associated with sodium stibogluconate therapy for American cutaneous leishmaniasis. Clin Infect Dis 1996; 22: 878-9.

17.- Jeddi F, Caumes E, Thellier M, Jauréguiberry S, Mazier D, Buffet P A. Drug hypersensitivity syndrome induced by meglumine antimoniate. Am J Trop Med Hyg 2009; 80: 939-40.

18.- Oliveira M C, Amorim R F, Freitas R de A, Costa A de L. A fatal case of mucocutaneous leishmaniasis after pentavalent antimonial use. Rev Soc Bras Med Trop 2005; 38: 258-60.

19.- España A, Hermida J M, Montilla P, Buzón L. Cutaneous and systemic leishmaniasis with a herpesvirus infection in an AIDS patient. Rev Clin Esp 1990; 187: 437.

20.- Barrio J, Lecona M, Cosin J, Olalquiaga F J, Hernanz J M, Soto J. Leishmania infection occurring in herpes zoster lesions in an HIV-positive patient. Br J Dermatol 1996; 134: 164-6.

21.- Llanos-Cuentas A, Echevarría J, Cruz M, La Rosa A, Campos P, Campos M, et al. Efficacy of sodium stibogluconate alone and in combination with allopurinol for treatment of mucocutaneous leishmaniasis. Clin Infect Dis 1997; 25: 677-84. 